\title{
Antiretroviral treatment efficacy after mutations reversion during T20 monotherapy, an alternative strategy in multi-failed HIV-I infected patients
}

\author{
S Lo Caputo*1, M Santoro², CSF Ceccherini-Silberstein², V Svicher ${ }^{2}$, \\ P Pierotti ${ }^{1}$, F Vichi ${ }^{1}$, R D'Arrigo ${ }^{3}$, C Gori ${ }^{3}$, CF Perno ${ }^{2}$ and F Mazzotta ${ }^{1}$
}

Address: ${ }^{1}$ S.M. Annunziata Hospital, Florence, Italy, ${ }^{2}$ University Tor Vergata, Rome, Italy and ${ }^{3}$ INMI - IRCCS - Lazzaro Spallanzani, Rome, Italy

* Corresponding author

from Ninth International Congress on Drug Therapy in HIV Infection

Glasgow, UK. 9-13 November 2008

Published: 10 November 2008

Journal of the International AIDS Society 2008, I I (SuppI I):P35 doi:I0.I I86/I758-2652-II-SI-P35

This abstract is available from: http://www.jiasociety.org/content/II/SI/P35

(c) 2008 Lo Caputo et al; licensee BioMed Central Ltd.

\section{Purpose of the study}

Monotherapy maintenance with 3TC after multiple therapeutic failure, helps in maintaining the number of CD4, but, at the same time, slows down the speed of reversion of mutations usually achieved during complete interruption of therapy. Monotherapy with enfuvirtide (T20) can be an interesting alternative to 3TC monotherapy, due to the CD4-enhancement typical of this drug even during therapeutic failure. Aim of this study was to assess, in a proof-of-concept study, the efficacy of T20-monotherapy to maintain the levels of CD4, to allow reversal of mutations in the pol gene, and eventually to favor long-term success of subsequent HAART.

\section{Methods}

In a pilot study named Reverfuzeon, four multi-failed HIV-infected patients with detectable viral load, nadir of CD4 $<200 / \mathrm{mmc}$, low compliance to a complex and longterm treatment and with $0-1$ fully active drug, were enrolled from 2004 to the beginning of 2006. All patients were on ARV for more than 10 years and all were exposed to NRTI, NNRTI and PI. Authors decided to establish a T20-monotherapy for a mean time of 180 days (range: 151-203) to achieve the reversion of all mutations expressed in pol gene maintaining an antiretroviral activity. Afterward patients started a new regimen containing $\mathrm{TDF}+\mathrm{FTC}+\mathrm{TPV} / \mathrm{r}$.

\section{Summary of results}

The mean CD4+ value before starting the T20-monotherapy was $308 / \mathrm{mmc}(190-554)$ and at the end was $241 /$ mmc (187-307), with a variation of CD4+ value of only $66 / \mathrm{mmc}$ in a 180 days average. Viral load did not show relevant deviations from a mean baseline value of 72,740 cps/ml (range: 2,500-270,000). Mutations during T20monotherapy decreased substantially for NRTI, NNRTI and PI from 5 (range: 4-7), 1.75 (0-3), 12.5 (9-14), respectively, to $1.25(0-4), 0.25(0-1), 4.75(1-9)$ after the end of T20-monotherapy assumption. Successively, after 12 months from starting the regimen containing $\mathrm{TDF}+\mathrm{FTC}+\mathrm{TPV} / \mathrm{r}$, plasma HIV-RNA was undetectable with an increase of $100 / \mathrm{mmc} C D 4+$. In three patients followed for $\geq 24$ months (min 24, $\max 40$ months) the viral load was still undetectable and a further increase of CD4+ was observed.

\section{Conclusion}

In this pilot study it was possible to build up an effective treatment where no other options were active at the moment of the study design, by reducing the number of resistance mutations. The T20-monotherapy resulted in a well tolerated treatment, from which to restart to obtain a virological success in multi-failed patients. 